\title{
Functionalized mesoporous silica nanoparticles for oral delivery of budesonide
}

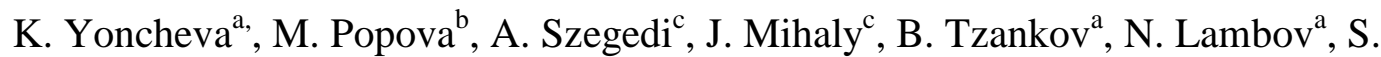 \\ Konstantinov $^{\text {a }}$, V. Tzankova ${ }^{\mathrm{a}}$, F. Pessina ${ }^{\mathrm{d}}$, M. Valotid ${ }^{\mathrm{d}}$
}
${ }^{a}$ Department of Pharmaceutical Technology, Faculty of Pharmacy, Medical University of Sofia, 2 Dunav Str., 1000 Sofia, Bulgaria
${ }^{\mathrm{b}}$ Institute of Organic Chemistry with Centre of Phytochemistry, Bulgarian Academy of Sciences, Sofia, Bulgaria
${ }^{c}$ Institute of Nanochemistry and Catalysis, Chemical Research Center, Hungarian Academy of Sciences, Pusztaszeri út. 59-67, 1025 Budapest, Hungary
${ }^{\mathrm{d}}$ Dipartimento di Scienze della Vita, Universita di Siena, via Aldo Moro 2, Siena, Italia

Keywords: Mesoporous silica nanoparticles, Budesonide release, Carbopol coating, Cytoprotective effect

\begin{abstract}
A b s t r a c t
Non-functionalized and amino-functionalized mesoporous silica nanoparticle were loaded with anti-inflammatory drug budesonide and additionally post-coated with bioadhesive polymer (carbopol). TEM images showed spherical shape of the nanoparticles and slightly higher polydispersity after coating with carbopol. Nitrogen physisorption and thermogravimetic analysis revealed that more efficient loading and incorporation into the pores of nanoparticles was achieved with the amino-functionalized silica carrier. Infrared spectra indicated that the post-coating of these nanoparticles with carbopol led to the formation of bond between amino groups of the functionalized carrier and carboxyl groups of carbopol. The combination of amino-functionalization of the carrier with the post-coating of the nanoparticles sustained budesonide release. Further, an in vitro model of inflammatory bowel disease showed that the cytoprotective effect of budesonide loaded in the post-coated silica nanoparticles on damaged HT-29 cells was more pronounced compared to the cytoprotection obtained with pure budesonide.
\end{abstract}




\section{Introduction}

Recently, research has been focused on the application of mesoporous silica based materials as a potential drug delivery system [1-5]. Mesoporous silica nanoparticles have high surface area and large pore volume that enable drug loading [6]. The mesoporous silica carriers could be functionalized with different organic groups (chloropropyl, phenyl, benzyl, mercaptopropyl, cyanopropyl, butyl groups) that additionally could improve their loading capacity [7-9]. Sulfadiazine possessing amino functional groups has been loaded in a higher degree in carboxyl functiona-lized silica carrier than in non-functionalized due to electrostatic binding [10]. Further, the formation of bonds could modify the release process, in particular could reduce the initial burst effect and could sustain the release rate of the incorporated drug [11-13]. For instance, erythromycin release from functionalized SBA-15 mesoporous silica was much slower compared to the release from unmodified material [11]. The surface modification of mesoporous silica nanoparticles with polymers is another approach intended for reduction of the initial burst release [14-17]. In addition, the surface modification could improve the interactions between nanoparticles and cells [18-20]. The mod-ification of mesoporous silica nanoparticles is an intensively developing field associated with some difficulties. For instance, drug loading into modified carriers could be hindered due to the partial blocking of the pore entrance. To overcome this drawback, drug loading could be performed at specific conditions, e.g. loading of doxorubicin in mesoporous carriers modified with poly (acrylic acid) was carried out in a buffer with $\mathrm{pH}$ of 7.4 to enable electrostatic interaction between negatively charged carboxyl groups of polymer and positively charged doxorubicin [21]. Another method for surface modification could be post-coating of mesoporous particles that are preliminary loaded with a drug.

Budesonide is a nonhalogenated glucocorticosteroid possessing potent local antiinflammatory activity that is used for the treat-ment of asthma, non-infectious rhinitis and inflammatory bowel diseases. A tendency towards an increase in inflammatory bowel diseases, among which ulcerative colitis and Crohn's disease, has been noticed worldwide [22]. Ulcerative colitis affects mainly the colon mucosa, whereas Crohn's disease affects the distal segments of the ileum. That is why the efforts in the recent years are pointed at preparation of appropriate budesonide delivery system with a local anti-inflammatory effect. However, the inflammation could affect gastrointestinal transit and drug absorption after oral administration. On the other hand, since budesonide has been identified as a P-glycoprotein substrate, its absorption could also be influenced by the P-glycoprotein efflux [23]. Thus, the efficient oral therapy with budesonide will depend on the capacity of drug delivery system to release budesonide in the distal parts of GIT (e.g. ileum and colon), and the ability to prolong its residence time in these regions [24]. One of the approaches that could prolong gastrointestinal residence is the incorporation of the drug into nanoparticles. In addition, depending on the properties of the nanoparticle carrier the release rate of budesonide could be adjusted.

In the present study, surface modification of the mesoporous nanoparticles was considered in 
order to provide low initial release and longer budesonide residence in gastrointestinal tract. The longer residence could be of a great importance taking into account that histological analyses showed high accumulation of silica nanoparticles in inflamed colon tissue [25]. For this reason, carbopol was selected as modifying agent mainly because of its capacity for strong bioadhesive interactions that could prolong drug residence. Further, formation of polymer layer around nano-particles could reduce the rapid burst release providing that high concentration of budesonide would reach the distal segments of the tract. The modification was invented as post-coating of the budesonide loaded particles because preliminary modification of the carriers could hinder drug loading into pores.

\section{Materials and methods}

\subsection{Materials}

Budesonide, 3-aminopropyltriethoxysilane (APTES), tetraethyl orthosilicate (TEOS) and Nhexadecyltrimethylammoniumbromide $\left(\mathrm{C}_{16} \mathrm{TMABr}\right)$ were purchased from Aldrich. HEP G2 and HT-29 cells were obtained from the German Collection of Microorganisms and Cell Cultures (DSMZ GmbH, Braunschweig, Germany). All other solvents and reagents were of analytical grade.

\subsection{Synthesis of MCM-41 mesoporous silica carrier}

MCM-41 was prepared according to the procedure of Huh et al. [26]. This sol-gel procedure is carried out at $353 \mathrm{~K}$ without a co-solvent, only in water solution and with $\mathrm{NaOH}$ as a catalyst. The relative molar composition of the reaction mixture was 1 TEOS: 0.12 $\mathrm{C}_{16}$ TMABr:0.31 NaOH:1190 $\mathrm{H}_{2} \mathrm{O}$. The formed gel was aged at $353 \mathrm{~K}$ for $2 \mathrm{~h}$, then washed with distilled water until neutral $\mathrm{pH}$, and dried at ambient. Template removal of MCM-41 materials was carried out in air at $823 \mathrm{~K}$ with $1 \mathrm{~K} / \mathrm{min}$ rate for $5 \mathrm{~h}$.

\subsection{Modification of the carrier by APTES}

Modification of the spherical MCM-41 material with amino groups was accomplished by reacting with 3-aminopropyltriethoxysilane (APTES) $(24 \mathrm{~h}, 333 \mathrm{~K})$ in toluene. After the reaction, the materials were washed with several portions of toluene, methanol and finally water. $1 \mathrm{~g}$ of the silica was reacted with $20 \mathrm{ml}$ APTES in $100 \mathrm{ml}$ toluene. The modified samples by APTES were designated as MCM-41 $\mathrm{NH}_{2}$. 


\subsection{Budesonide loading and post-coating of the mesoporous nanoparticles}

Mesoporous carriers were incubated with budesonide in ethanol under continuous magnetic stirring for $24 \mathrm{~h}$ at $310 \mathrm{~K}$. Briefly, $100 \mathrm{mg}$ of budesonide was dissolved in $2.5 \mathrm{ml}$ of ethanol and $100 \mathrm{mg}$ of dried mesoporous silica was put into this solution. After $24 \mathrm{~h}$, the mixture was centrifuged at 15,000 rpm, rinsed with purified water, separated by a second centrifugation, and finely dried at room temperature under vacuum.

For the coating procedure, drug loaded particles were incubated in an aqueous phase containing carbopol $(0.012 \mathrm{wt} \%)$ under gentle stirring $(100 \mathrm{rpm})$ for $2 \mathrm{~h}$. After incubation, the dispersions were centrifuged at 15,000 rpm for $15 \mathrm{~min}$, rinsed with distilled water, separated by a second centrifugation, and finely dried at room temperature under vacuum. The amount of budesonide in the supernatants was measured by UV-spectrophotometry at a wavelength of $250 \mathrm{~nm}$ (Hewllet Packard 8452 A). The concentration of budesonide was calculated according to the standard curve prepared in the concentration range of 7-28 $\mu \mathrm{g} / \mathrm{ml}(\mathrm{r}>0.994)$. The samples were abbreviated as MCM-41budC and MCM- $41 \mathrm{NH}_{2}$ budC.

\subsection{Characterization of the nanoparticles}

X-ray diffractograms were recorded by a Philips PW 1810/3710 diffractometer with BreggBrentano parafocusing geometry apply-ing monochromatized $\mathrm{CuK}_{\alpha}(\lambda=0.15418 \mathrm{~nm})$ radiation $(40 \mathrm{kV}, 35 \mathrm{~mA})$ and proportional counter.

Nitrogen physisorption measurements were carried out at $77 \mathrm{~K}$ using Quantachrome NOVA Automated Gas Sorption Instrument. The pore-size distributions were calculated from the desorption branch of the isotherms with the BJH method. Samples were pretreated at 353 $\mathrm{K}$ for $5 \mathrm{~h}$ before measurements.

TEM images were taken by using a MORGAGNI 268D TEM (100 kV; W filament; pointresolution $0.5 \mathrm{~nm})$.

Attenuated total reflection infrared (ATR-FTIR) spectra were recorded by means of a Varian Scimitar 2000 FTIR spectrometer equipped with a MCT (mercury-cadmium-tellur) detector and a single reflection ATR unit (SPECAC "Golden Gate") with a diamond ATR element. In general, 128 scans and $4 \mathrm{~cm}^{-1}$ resolution were applied. For all spectra ATR-correction was performed (Varian ResPro 4.0 software).

Determinations of nanoparticle size, polydispersity index and zeta potential were performed using a Zetasizer (Zetasizer Nano ZS, Malvern Instruments, Worcestershire, UK). The samples were dispersed in distilled water and measured at a scattering angle of $90^{\circ}$ and $25^{\circ}$.

The budesonide loading was determined by thermogravimetric measurements performed with a Setaram TG92 instrument with a heating rate of $5 \mathrm{~K} / \mathrm{min}$ in nitrogen flow. 


\subsection{In vitro release studies}

For in vitro release studies, $10 \mathrm{mg}$ of the drug loaded nanoparticles were incubated in $200 \mathrm{ml}$ acid or phosphate buffer $(\mathrm{pH}=1.2$ or 6.8$)$ at $310 \mathrm{~K}$ under stirring (100 rpm). Samples were withdrawn $(5 \mathrm{ml})$ at appropriate time intervals, centrifuged at $15,000 \mathrm{rpm}$ for $15 \mathrm{~min}$, and replaced by fresh buffer ( $\mathrm{pH}=1.2$ or 6.8$)$. The concentration of the released budesonide in the samples was deter-mined by UV-vis spectrophotometry at a wavelength of $250 \mathrm{~nm}$ (Hewllet Packard 8452 A) as described above.

\subsection{In vitro cytotoxicity}

The HEP G2 were placed into $75 \mathrm{~cm}^{2}$ tissue culture flasks and grown at $310 \mathrm{~K}$ under a humidified $5 \% \mathrm{CO}_{2}$ atmosphere in 90\% DMEM with $2 \mathrm{mM}$ glutamine containing $1.5 \mathrm{~g} / \mathrm{l}$ sodium bicarbonate and supplemented with $10 \%$ fetal bovine serum and $2 \%$ penicillinstreptomycin (10,000 U/ml penicillin and $10 \mathrm{mg} / \mathrm{ml}$ streptomycin) (Gibco BRL).

HEP G2 cells were placed in a 96-well plate at a density of $2 \times 10^{5}$ cells/well and allowed to attach to the well surface for $24 \mathrm{~h}$ at $310 \mathrm{~K}, 5 \% \mathrm{CO}_{2}$ and $95 \%$ air. Stock solutions of pure budesonide, empty and loaded carriers were freshly prepared in ethanol and thereafter diluted with growth medium to the desired concentrations (ethanol final concentration did not exceed $0.2 \%$ ). Different amounts (in the range of $0.1-1 \mathrm{mg} \mathrm{ml}^{-1}$ ) of budesonide loaded mesoporous silica particles were added to cells, and incubated for periods of $24 \mathrm{~h}$ and $48 \mathrm{~h}$. Eight wells for each concentrations were used. To determine cell viability, lactate dehydrogenase leakage (LDH) from the cells was determined using a commercial LDH cytotoxicity detection kit according to the manufacturer's protocols (Clontech, USA). Briefly, LDH activity is determined by colorimetric assay. In the first step, NAD+ is reduced to $\mathrm{NADH} / \mathrm{H}+$ by the LDH-catalyzed conversion of lactate to pyruvate. Then, a catalyst induced in the reaction mixture (diaforase) transfers $\mathrm{H} / \mathrm{H}+$ from $\mathrm{NADH} / \mathrm{H}+$ to the tetrazolium salt INT, which is reduced to a formazan dye. The amount of dye produced is proportional to the number of lysed (dead or plasma membrane damaged) cells. The formazan dye product shows an absorbance maximum at $490 \mathrm{~nm}$. LDH activity was assessed in the conditioned media and the amounts detected were calculated as percentage of the solvent treated control $(0.2 \%$ ethanol in DMEM growth medium) (GraphPadPrizm Software).

\subsection{In vitro cytoprotective effect of budesonide loaded silica particles}

EOL-1 (ACC 386) cells originated from a patient with acute myeloid (eosinophilic) leukemia. HT-29 cells (human colon adenocarcinoma cell line) were obtained from the German Collection of Microorganisms and Cell Cultures (DSMZ GmbH, Braunschweig, Germany). For the study, EOL-1 and HT-29 cells were placed in the microplates at a density of $3 \times 10^{5}$ 
cells per $\mathrm{ml}$ and $1.5 \times 10^{5}$ per ml, respectively. After a $24 \mathrm{~h}$ incubation period at $310 \mathrm{~K}$, EOL1 cells were treated with $100 \mu \mathrm{g} / \mathrm{ml}$ phorbol 12-myristate 13-acetate (PMA) for $24 \mathrm{~h}$ and later were admixed to a HT-29 confluent monolayer. HT-29 cells were untreated (control) or pretreated with pure budesonide $(5$ or $10 \mu \mathrm{M})$ or budesonide loaded nano-particles. After $48 \mathrm{~h}$ incubation, the cells were rinsed with PBS and $3 \mathrm{ml}$ of new medium (RPMI 1640) and $30 \mu \mathrm{l}$ MTT solution were added to each well. The microplates were further incubated for $3 \mathrm{~h}$ at 310 $\mathrm{K}$ and the MTT-formazan crystals formed were dissolved through the addition of $3 \mathrm{ml}$ per well of 5\% formic acid in 2-propanol. The MTT formazan absorption was determined using a microprocessor controlled microplate reader (Labexim LMR-1) at $550 \mathrm{~nm}$.

\subsection{Statistical analysis}

The experiments were replicated three independent times and the data are presented as mean \pm SEM. Statistical analysis was carried out using Student's t test. Differences were considered statistically significant when P-value was less than 0.01.

\section{Results and discussion}

In this study amino-functionalization of mesoporous silica nanoparticles was performed in order to provide available functional groups for subsequent coating with carbopol. The coating was intended in order to reduce the burst release of budesonide as well as to achieve long residence of budesonide on intestinal mucosa due to bioadhesive properties of carbopolcoated mesoporous nanoparticles.
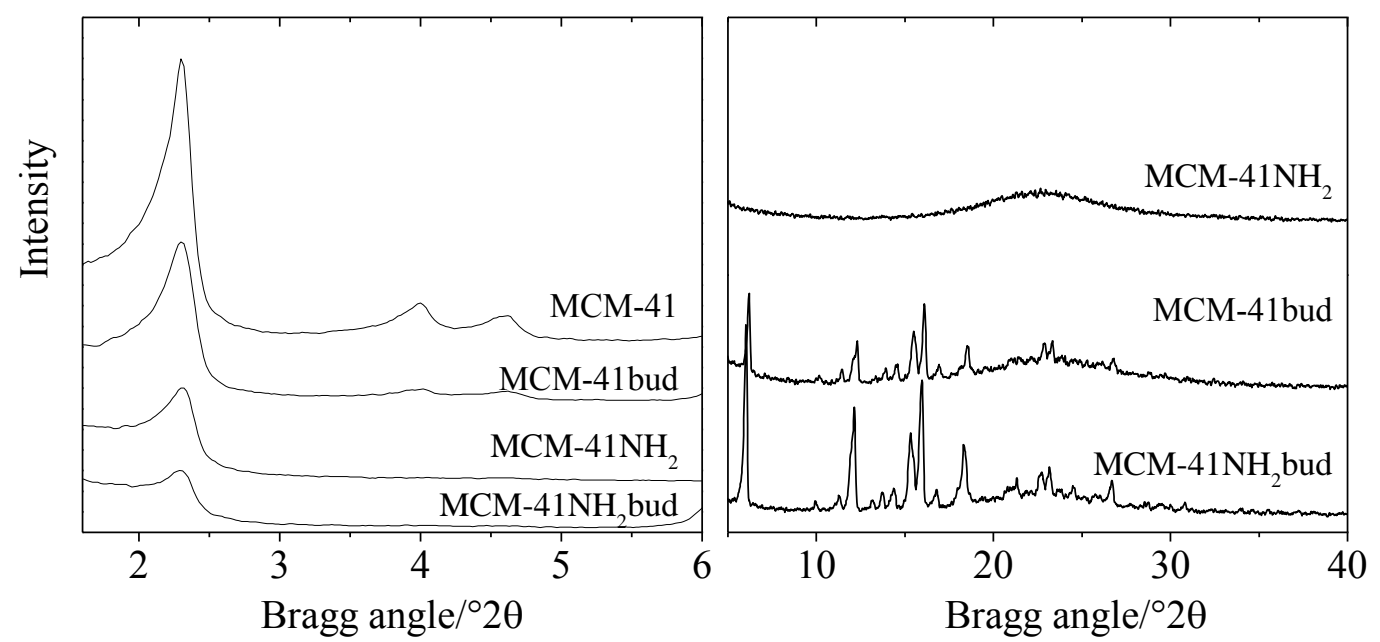

Fig. 1. XRD patterns of initial (MCM-41) and amino-functionalized (MCM-41NH $\left.{ }_{2}\right)$ mesoporous particles 


\subsection{Physicochemical characterization of the mesoporous nanoparticles}

XRD data of the initial (MCM-41) and the amino-functionalized (MCM-41NH $\mathrm{N}_{2}$ samples with the intense (100) and higher Miller indices reflections in the low 2 theta region confirmed the formation of the hexagonal structure ( Fig. 1). Some intensity decrease and broadening of the 100 reflection are observed in the pattern of amino-functionalized MCM-41 indicating partial structure deterioration. Broadening and shifting of the diffraction peaks were observed for the budesonide loaded samples, especially for the amino-functionalized one. This effect indicated more significant decrease in structural order in the case of functionalized drug loaded sample, which could be related to higher drug loading capacity. Also, the presence of crystalline budesonide phase was registered on the non-functionalized and the amino-functionalized samples. The latter evidenced that budesonide was not only contained in the mesopore channels, but could be found on the outer surface of the spherical particles or in the secondary mesopores, e.g. in the voids among the particles.

Nitrogen adsorption and desorption isotherms of the samples are presented in Fig. 2 whereas the calculated textural parameters are presented in Table 1 . The isotherms of the initial and the amino-functionalized samples exhibited a sharp increase at a relative pressure of $\mathrm{p} / \mathrm{p}_{0}=$ $0.2-0.4$, which was associated with capillary condensation in the channels and narrow pore size distribution ( Fig. 2). The isotherms of the MCM-41 samples were reversible and did not show any hysteresis loop. The textural parameters of budesonide loaded non-functionalized particles showed some decrease in the surface area, pore diameter and pore volume. The modification by APTES resulted in some decrease of the surface area, pore diameter and pore volume whereas the budesonide loading on functionalized $\mathrm{MCM}-41 \mathrm{NH}_{2}$ carrier led to a total pore filling. The prepared silica MCM-41 support is made up of $100 \mathrm{~nm}$ sized spherical particles, which can agglomerate and among them secondary mesopores are formed. These mesopores are much bigger than the channel system of the particles but also suitable to store the drug. The hysteresis loop of capillary condensation of nitrogen into these secondary mesopores at relative pressures near $\mathrm{p} / \mathrm{p}_{0}=1$ is observed for all samples ( Fig. 2).

All mesoporous samples were characterized by FT-IR, verifying the presence of amino groups after functionalization and drug adsorption. The asymmetric stretching vibrations ( $\mathrm{Si}-$ O-Si) appeared at about $1090 \mathrm{~cm}^{-1}$ ( Fig. 3). The modification of MCM-41 samples by APTES resulted in the appearance of the bands at $2929 \mathrm{~cm}^{-1}$ and at $1540 \mathrm{~cm}^{-1}$, which was attributed to $\mathrm{C}-\mathrm{H}$ and $\mathrm{N}-\mathrm{H}$ stretching vibrations of aminopropyl anchored on the surface of the mesoporous carrier [17]. According to the literature, primary aliphatic amines absorb in the region 3450-3160 $\mathrm{cm}^{-1}$, resulting in a broad band of medium intensity [27]. In solid state, amine hydrohalides containing $-\mathrm{NH}_{3}{ }^{+}$have an absorption of medium intensity at 3350-3100 $\mathrm{cm}^{-1}$. Primary amines have a medium-to-strong absorption band in the $1650-1580 \mathrm{~cm}^{-1}$ region. For $-\mathrm{CH}_{2} \mathrm{NH}_{3}{ }^{+}$functional group, the asymmetric $\mathrm{NH}_{3}{ }^{+}$deformation appears at 1615$1560 \mathrm{~cm}^{-1}$ as a medium-to-strong band, while the symmetric $\mathrm{NH}_{3}{ }^{+}$deformation appears around $1520-1480 \mathrm{~cm}^{-1}$ as a weak intensity band [27]. 

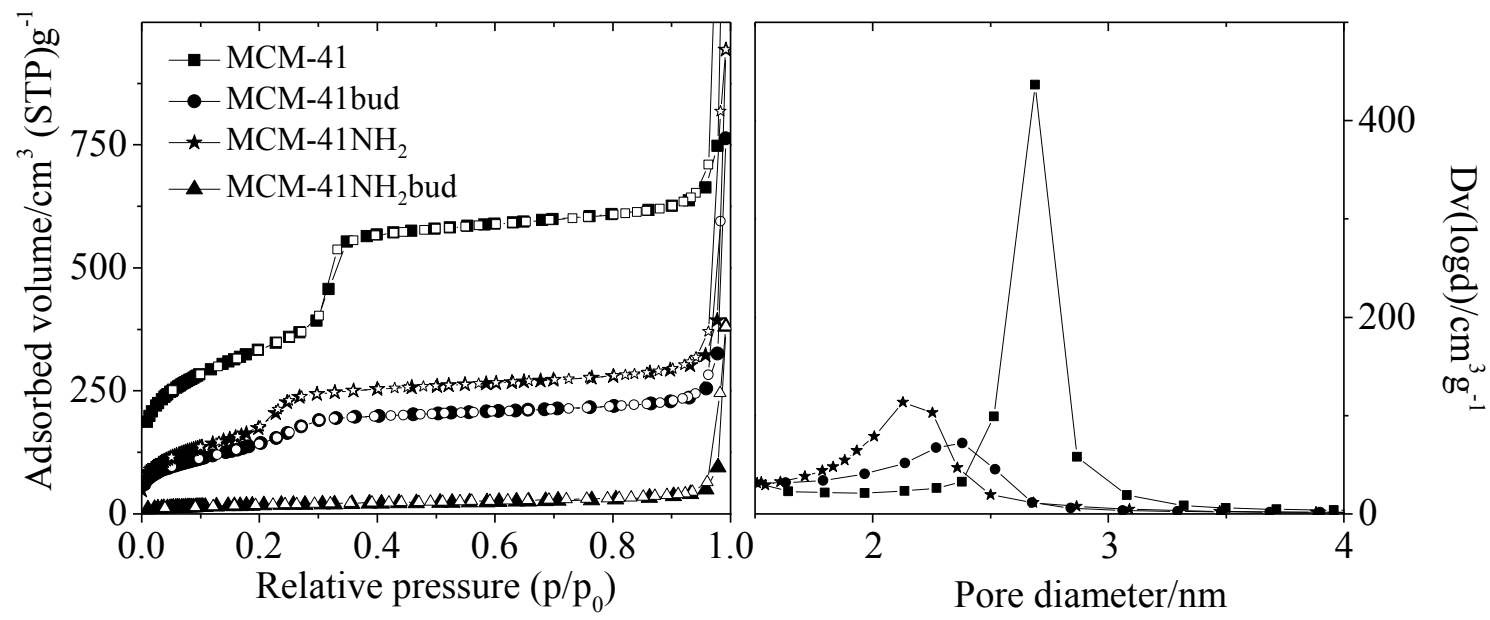

Fig. 2. Nitrogen adsorption/desorption isotherms of initial (MCM-41) and aminofunctionalized (MCM-41NH 2$)$ mesoporous particles.

Table 1

Physico-chemical properties of the initial and amino-functionalized particles.

\begin{tabular}{|c|c|c|c|c|c|}
\hline Samples & $\begin{array}{l}\mathrm{a}_{\mathrm{o}}^{\mathrm{a}} \\
(\mathrm{nm})\end{array}$ & $\begin{array}{l}\text { BET } \\
\left(\mathrm{m}^{2} / \mathrm{g}\right)\end{array}$ & $\begin{array}{l}\mathrm{PD}^{\mathrm{b}} \\
(\mathrm{nm})\end{array}$ & $\begin{array}{l}\text { Pore } \\
\text { volume }\left(\mathrm{cm}^{3} / \mathrm{g}\right)\end{array}$ & $\begin{array}{l}\text { Zeta } \\
\text { potential }(\mathrm{mV})\end{array}$ \\
\hline MCM-41 & 4.43 & 1175 & 2.7 & 0.99 & $-12.17 \pm 1$ \\
\hline MCM-4141NH 2 & 4.42 & 570 & 2.2 & 0.47 & $+34.17 \pm 2$ \\
\hline MCM-41Bud & 4.42 & 473 & 2.4 & 0.40 & $-14.67 \pm 1$ \\
\hline $\mathrm{MCM}-41 \mathrm{NH}_{2} \mathrm{Bud}$ & 4.45 & 62 & n.d. & 0.16 & $+35.37 \pm 3$ \\
\hline
\end{tabular}

${ }^{\mathrm{a}}$ Cell parameter $\left(\mathrm{a}_{\mathrm{o}}=2 \mathrm{~d}_{100}(3)^{-1 / 2}\right)$.

${ }^{\mathrm{b}}$ Pore diameter and pore volume calculated by BJH method.

The FT-IR spectra of budesonide loaded particles showed the bands at 2962 and $1463 \mathrm{~cm}^{-1}$ characteristic for $\mathrm{C}-\mathrm{H}$ and phenyl bands, respectively. The presence of the band at $1555 \mathrm{~cm}^{-1}$ in the spectra of the carbopol-coated amino-functionalized silicas was indicative for the formation of a $\mathrm{COO}^{-}-\mathrm{NH}_{3}{ }^{+}$bond between the polymer and amino groups on the outer surface of the carrier. In the case of carbopol-coated non-functionalized particles interaction between carboxyl groups of carbopol and silanol groups of the carrier was not observed.

The zeta potential of the non-functionalized MCM-41 particles was negative whereas the positive value was measured for particles prepared with amino-functionalized carrier (MCM$41 \mathrm{NH}_{2}$ ). These observations proved the successful functionalization and the presence of amino groups on the surface ( Table 1). The drug loading did not influence the surface charge of the amino-functionalized particles, which was an indication for the availability of the amino groups on the mesoporous surface for further interaction with the coating polymer. 


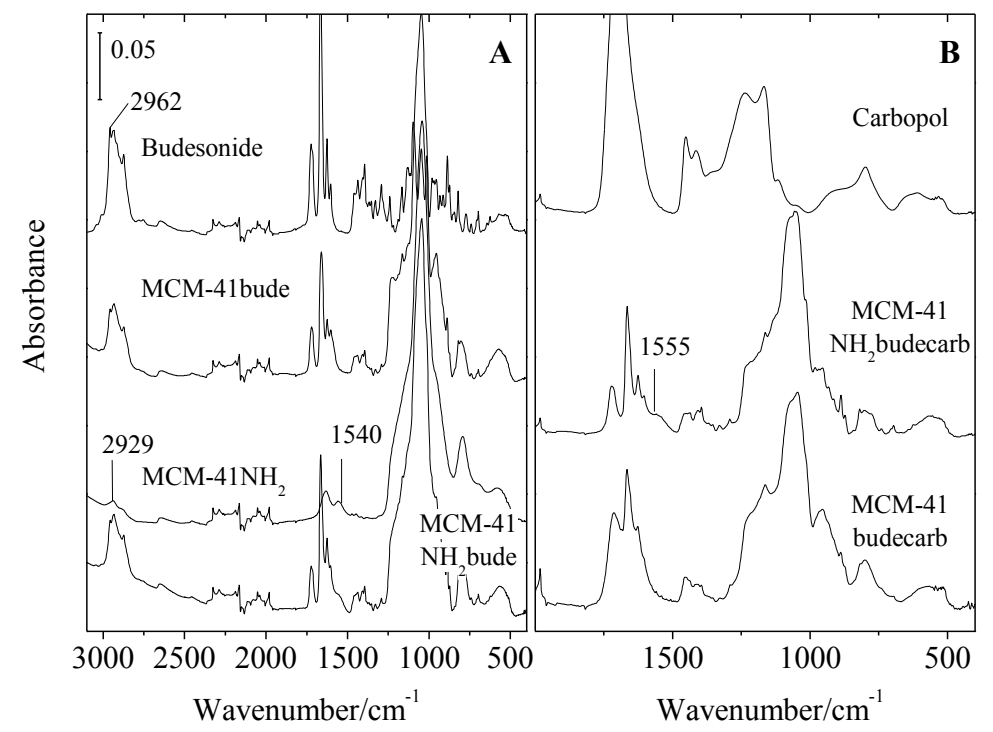

Fig. 3. ATR-FTIR spectra of initial (MCM-41) and amino-functionalized (MCM-41NH $\left.\mathrm{N}_{2}\right)$ mesoporous particles.
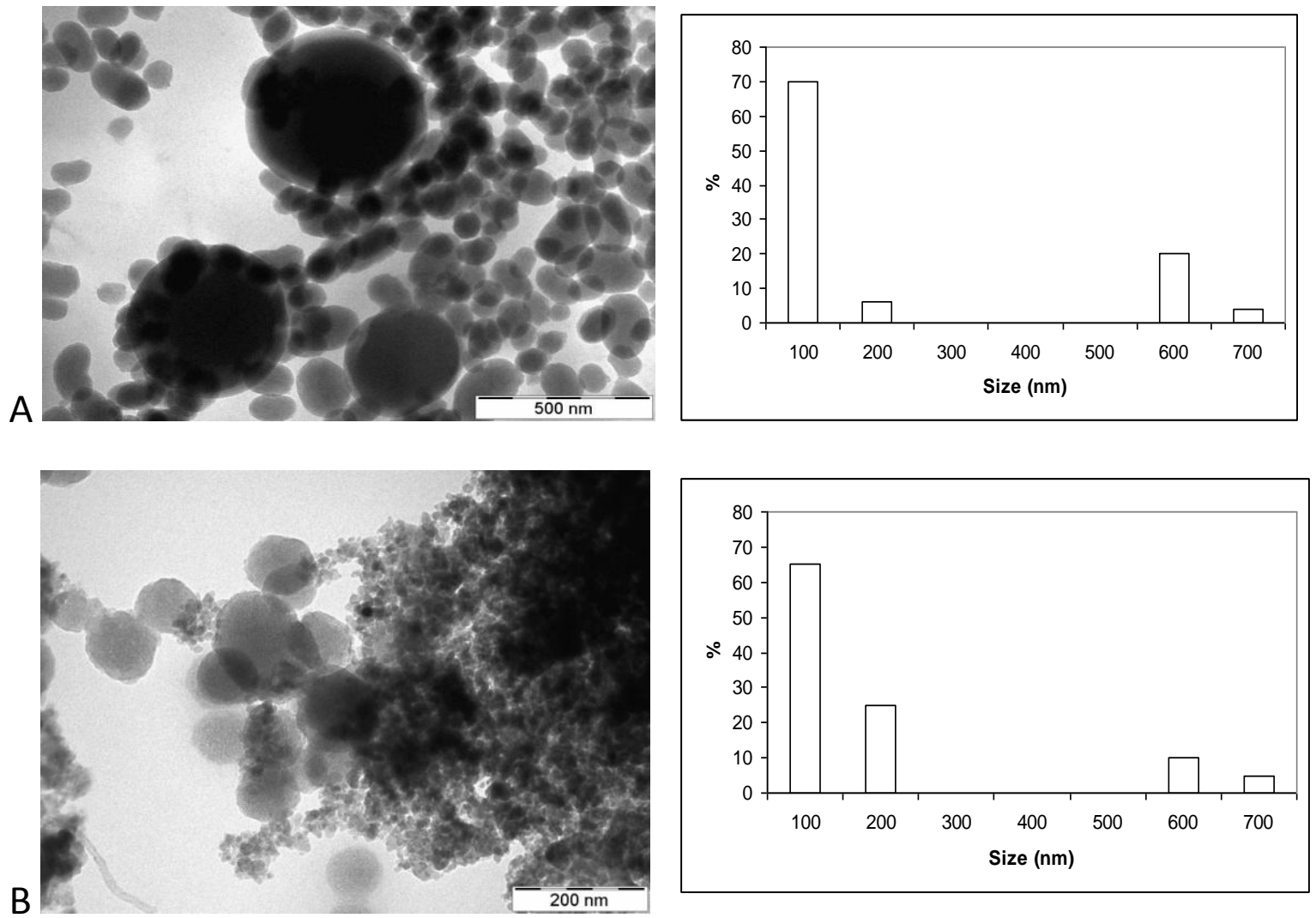

Fig. 4 TEM images and particle size distribution of carbopol-coated mesoporos particles: MCM-41budC (A) and MCM-41NH2budC (B). 
A

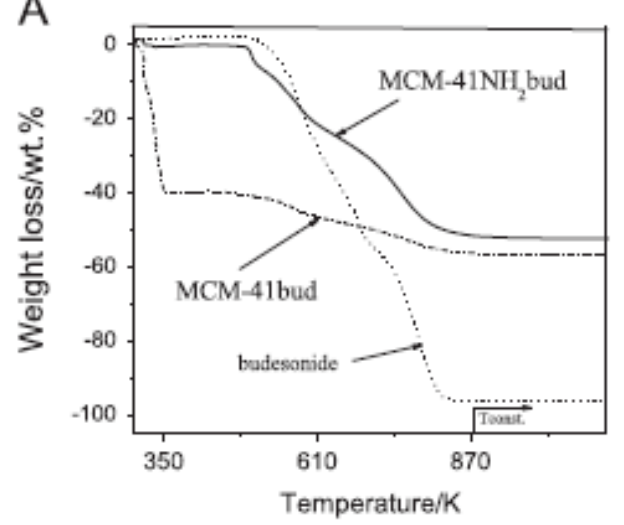

B

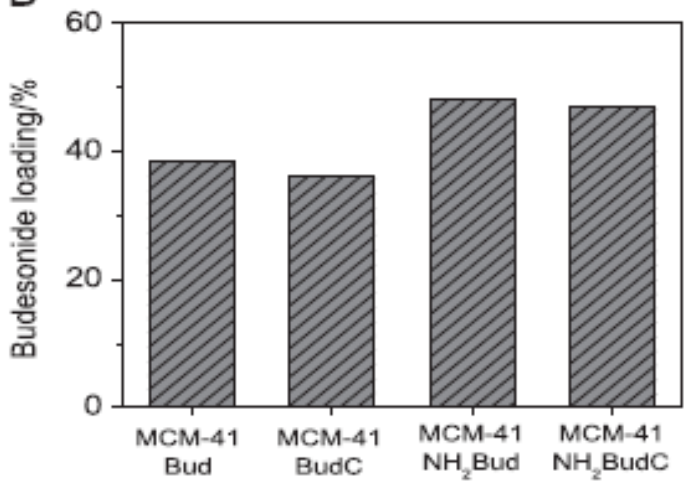

Fig. 5. Thermogravimetric profiles of initial and amino-functionalized budesonide loaded nanoparticles (MCM-41bud and $\mathrm{MCM}-41 \mathrm{NH}_{2}$ bud) (A) and comparison of budesonide loading into the different nanoparticles (B).

Determination of the size of budesonide loaded carbopol-coated particles showed that a fraction with a size $100 \mathrm{~nm}$ was about 70\%, which was in a good accordance with TEM images ( Fig. 4).

The budesonide loaded in the mesopores of the nanoparticles and pure budesonide were quantified using thermogravimetry (TG). The calculated amount of amino groups in the amino-functionalized MCM- $41 \mathrm{NH}_{2}$ is around $2.5 \mathrm{wt} \%$. The actual amount of the drug loading was determined after correcting the curves by water and aminopropyl content. The nonfunctionalized MCM-41 showed lower adsorption capacity (40 wt\%) for budesonide in comparison to its amino-functionalized analog (49 wt\%) ( Fig. 5). Thermogravimetric profile of non-functionalized particles showed that about $71 \%$ of budesonide was decomposed in the interval 573-623 K, indicating that a significant part of budesonide was physically adsorbed outside the channels of non-functionalized carrier, probably in the secondary mesopores ( Fig. 5A). The decomposition of non-loaded budesonide ( Fig. 5A) started at higher temperature than the loaded one. This observation is typical when the drug is finely dispersed on the support. The amount of budesonide loaded in the channels (decomposing above $500 \mathrm{~K}$ ) was relatively lower $(29 \%)$, whereas the whole amount of budesonide loaded on aminofunctionalized MCM-41 $\mathrm{NH}_{2}$ was decomposed above $480 \mathrm{~K}$. These results suggested that more efficient loading into the pores was achieved with the amino-functionalized carrier which is in a good accordance with the registered broadening and shifting of the diffraction peaks in their XRD patterns. This fact was explained with probable formation of hydrogen bonds between hydroxyl groups of budesonide and amino groups of the functionalized carrier. The coating of the drug loaded particles was not expected to influence the drug loading degree. Indeed, thermogravimetric measurements revealed similar loading for coated mesoporous nanoparticles with those for their non-coated analogs ( Fig. 5B). This observation was proved by the fact that during the coating procedure budesonide did not diffuse out from the particles probably due to the high viscosity of the coating solution. 

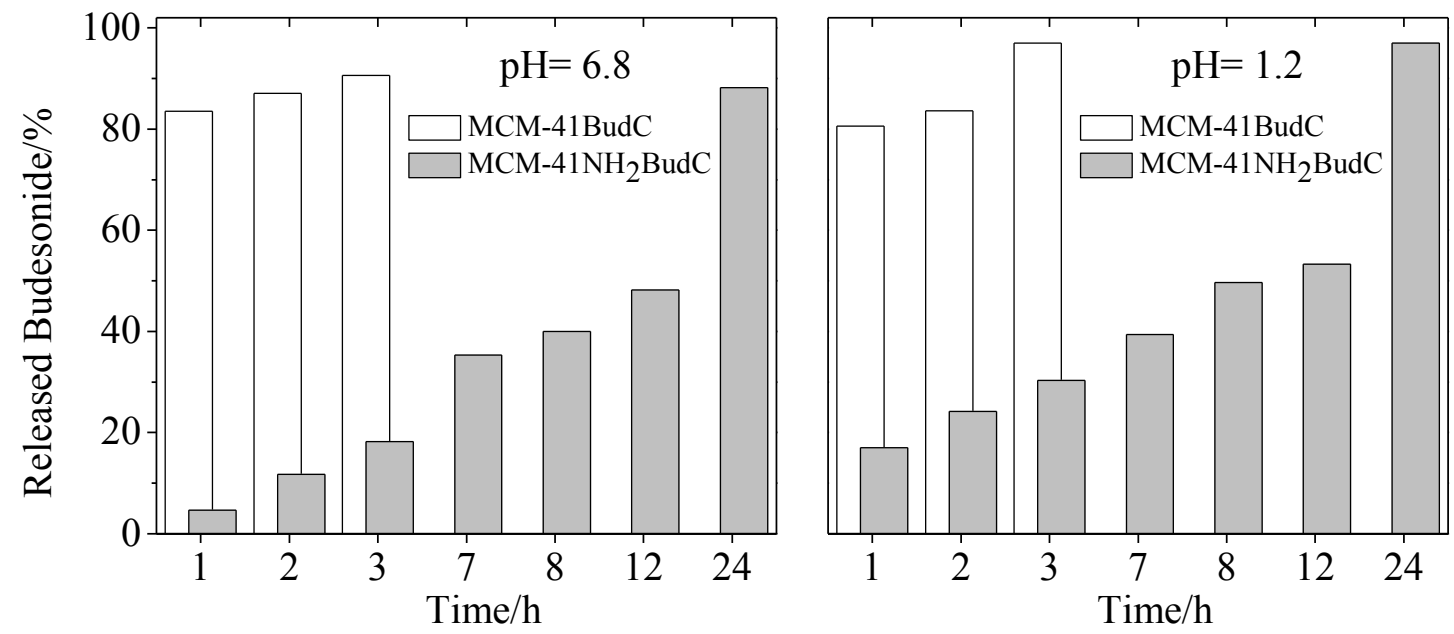

Fig. 6. In vitro budesonide release from carbopol-coated MCM-41budC (empty columns) and carbopol-coated amino-functionalized $\mathrm{MCM}-41 \mathrm{NH}_{2}$ budC (full columns) at $\mathrm{pH}=1.2$ and $\mathrm{pH}=6.8$.

\subsection{Drug delivery}

Release studies were performed in buffers with $\mathrm{pH}$ values 1.2 and 6.8 that are typically applied in order to mimic the in vivo conditions in gastrointestinal tract. The studies showed different profiles for coated and non-coated nanoparticles. Total budesonide release from noncoated nanoparticles was registered in $1 \mathrm{~h}$ at both $\mathrm{pH}$ values (not shown). Thus, independent on the fact that almost the whole amount of budesonide was located into the pores of the amino-functionalized carrier the release was as fast as from non-functionalized carrier where a significant part of budesonide was adsorbed outside the pores. Further, the coating of particles prepared with non-functionalized carrier resulted in negligible prolongation ( Fig. 6). However, the combination of amino-functionalization of the carrier with the post-coating of the particles really sustained the release. As shown, $100 \%$ drug was released in $3 \mathrm{~h}$ from coated non-functionalized nanoparticles, whereas from the coated functionalized nanoparticles the drug was released in $24 \mathrm{~h}$. The faster release from the coated nonfunctionalized particles was explained with the lower stability of the coating layer. Since the FTIR spectra did not reveal formation of bonds between silanol groups and carboxyl groups of the carrier, the surface layer was most probably formed by simple physical adsorption. More stable coating layer was formed with amino-functionalized carrier because of the $\mathrm{COO}^{-}$ $-\mathrm{NH}_{3}{ }^{+}$bond between carboxyl groups of carbopol and the amino groups of the carrier as FTIR spectra showed.

Drug loading degree could also influence the release process. However, the particles with similar loading released budesonide in different rates. In particular, non-coated and carbopol- 
coated amino-functionalized particles had similar load of approximately $49 \%$ but different release rates. Consequently, drug release was influenced mainly by the coating rather than different drug loadings.

The sustained release of budesonide was more pronounced at $\mathrm{pH}=6.8$. The carbopol shell around amino-functionalized particles at acidic conditions will be protonated that facilitates the drug release whereas at high $\mathrm{pH}$ value an extended shell around the particles will be formed. This extended shell could hinder the budesonide release from the silica core at pH=6.8 ( Fig. 6). Song et al. [28] prepared $\mathrm{pH}$ responsive membranes by grafting poly (acrylic acid) onto the silica-aluminum. The authors found high water permeation at low $\mathrm{pH}$, which is in good agreement with our observations.

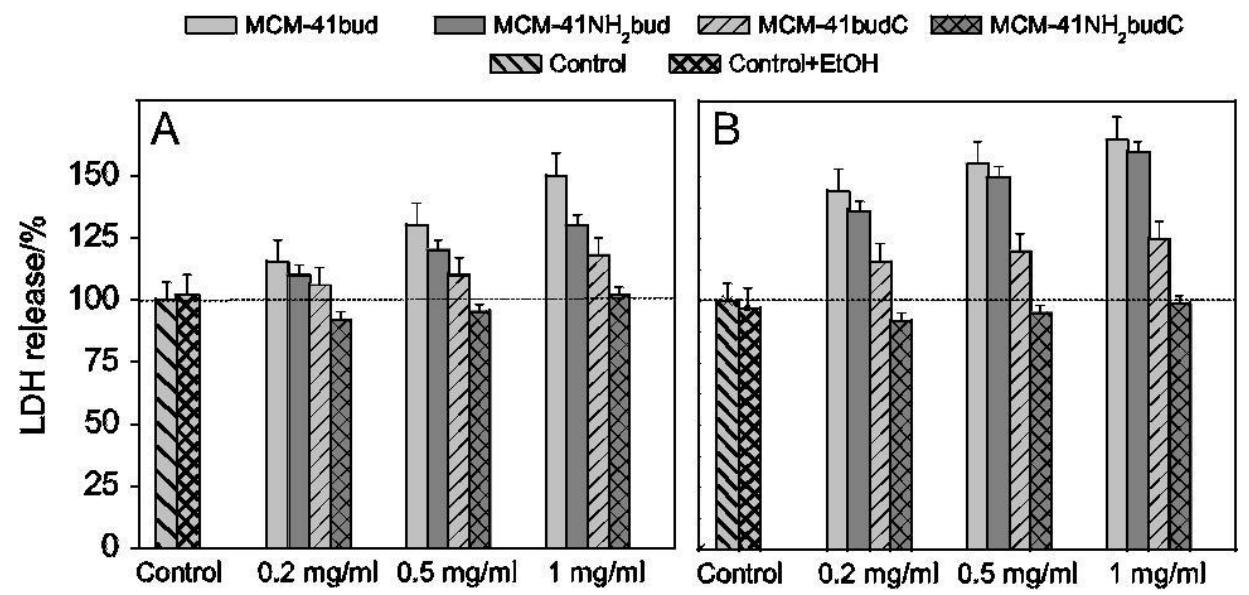

Fig. 7. LDH release from HEP G2 cells treated with the budesonide loaded mesoporous silica nanoparticles: $24 \mathrm{~h}$ and $48 \mathrm{~h}$ exposure (A and $\mathrm{B}$, respectively). Mean $\pm \mathrm{SD}(\mathrm{n}=3)$.

\subsection{In vitro cytotoxicity}

HEP G2 cells were selected for the study since they resemble functionally and morphologically normal hepatocytes. The in vitro cytotoxicity of the mesoporous nanoparticles was measured in HEP G2 cells using lactate dehydrogenase (LDH) assay to evaluate the integrity of cell membrane. First, the ability of the carriers and free budesonide to interfere with the cell membrane integrity was studied. Both the carriers and budesonide were practically devoid of cytotoxic effect by means of their LDH leakage (data not shown). Further, budesonide loaded non-coated particles (MCM-41Bud and MCM-41NH ${ }_{2} \mathrm{Bud}$ ) were incubated on HEP G2 cells and LDH leakage was determined in $24 \mathrm{~h}$ and $48 \mathrm{~h}$. After $24 \mathrm{~h}$ incubation time, both types of the particles showed an increase in LDH leakage suggesting changes in cell integrity. The increase was more prominent with the higher dose tested (1 $\mathrm{mg} / \mathrm{ml}$ ) (by $50 \%$ and by $30 \%$ vs control, p<0.01, respectively) ( Fig. 7A). After 48 h exposure 


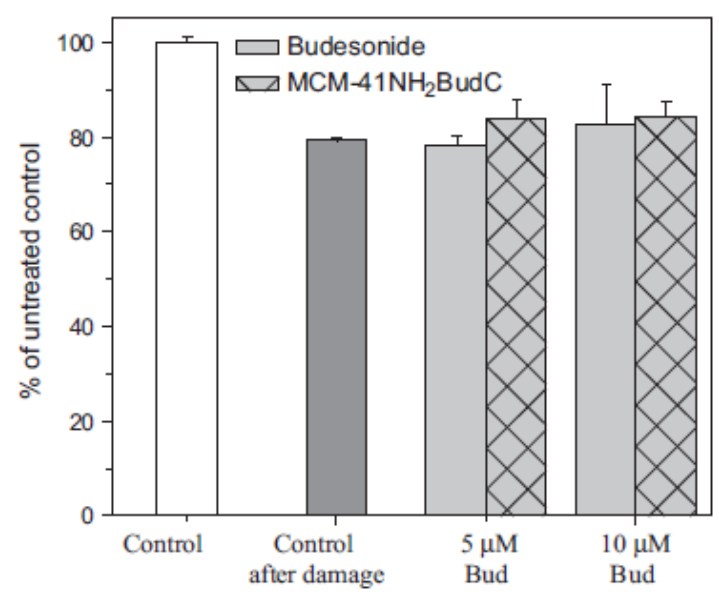

Fig. 8. Cytoprotective effect of free budesonide and budesonide loaded mesoporous silica nanoparticles (MCM-41NH 2 budC) on HT-29 cells damaged with phorbol-12-myristate-13acetate. Mean $\pm \operatorname{SD}(\mathrm{n}=3)$.

at varying doses, HEP G2 cells viability resulted in adose-dependent decrease as detected by LDH leakage ( Fig. 7B). However, the viability of the cells incubated with the coated mesoporous particles was different. Both types of carbopol-coated particles (MCM-41BudC and MCM-41 $\mathrm{NH}_{2} \mathrm{BudC}$ ) did not show statistically significant increase in LDH release (by $18 \%$ and by $2 \%$ vs. control, respectively). They were practically non-cytotoxic in the model of cellular population used in the study. Therefore, the coating of mesoporous silica with carbopol decreased the toxicity of the mesoporous drug delivery system.

\subsection{Cytoprotective effect}

In order to create an in vitro model of inflammatory bowel disease human mixed mononuclear cells (MMCs) were cocultured with HT-29 cells [29]. HT-29 cells (human colon adenocarcinoma cell line) can be used as in vitro model of intestinal mucosa because they have the characteristics of normal intestinal epithelium such as epithelial polarity, presence of the actin-binding protein villin, and the occurrence of an enterocytic differentiation [30]. EOL-1 cells are known to respond to phorbol myristate acetate ester (PMA) treatment and they provide a useful experimental model for the study of differentiation and regulation of human eosinophils. Moreover, EOL-1 cells are suitable as an in vitro eosinophilic model for studying eosinophilic functions [31,32].

Taking into account these considerations, PMA differentiated human EOL-1 cells were applied in a co-cultural model with HT-29 cells untreated or pretreated with pure budesonide or budesonide loaded nanoparticles. The addition of PMA treated EOL-1 cells caused about $20 \%$ decrease of the cell viability of monolayer growing colon HT-29 cells. Pretreatment of HT-29 cells with $5 \mu \mathrm{M}$ free budesonide did not protect cells from cytotoxic damage ( Fig. 8). The higher applied concentration of budesonide $(10 \mu \mathrm{M})$ provided cytoprotective effect. More 
interestingly, the pretreatment with budesonide loaded silica nanoparticles (MCM$41 \mathrm{NH}_{2}$ budC) caused significant protection against the damage produced by PMA differentiated eosinophilic EOL-1 cells ( Fig. 8). Even the lower concentration of nanoparticle formulation (corresponding to $5 \mathrm{mM}$ budesonide) resulted in statistically significant cytoprotective effect. Thus, the important finding was that the cytoprotection could be achieved at lower concentration of budesonide when loaded in mesoporous silica nanoparticles. Similarly, PEG-modified mesoporous silica nanoparticles loaded with hydralazine showed higher neuroprotective effect to PC12 neuronal cells damaged by exposure to acrolein than free hydralazine [33]. Controlled release of hydralazine and enhanced intracellular internalization were considered among the reasons for the significantly higher neuro-protective effect achieved with the mesoporous nanoparticles. In our study, the fact that the cytoprotection could be achieved at lower concentration by applying encapsulated budesonide could be explained with facilitated interaction of mesoporous nanoparticles with the cells.

\section{Conclusion}

Amino-functionalization of mesoporous silica particles resulted in higher loading degree compared to non-functionalized samples. On the other hand, the functionalization enabled the achievement of surface coating based on the interaction between amino groups of the carrier and carboxyl groups of carbopol. Both, the amino-functionalization and the coating, provided sustained release of budesonide during $24 \mathrm{~h}$ without initial burst effect. These particles provided cytoprotective effect on damaged model of intestinal mucosa at lower concentration than respective concentration of free budesonide. Thus, the development of modified silica nanoparticles loaded with budesonide could be considered as an alternative approach in the treatment of intestinal bowel diseases.

\section{Acknowledgments}

Financial support by the Bulgarian-Hungarian Inter-academic Exchange Agreement is greatly acknowledged. The work was also supported by Medical University of Sofia, Contract no. 1$\mathrm{D} / 2012$.

\section{References}

[1] J. Lu, M. Liong, J. Zink, F. Tamanoi, Small 3 (2007) 1341-1346.

[2] I. Slowing, J. Vivero-Escoto, C.W. Wu, V.S.-Y. Lin, Adv. Drug Deliv. Rev. 60 (2008) $1278-1288$.

[3] S. Wang, Micropor. Mesopor. Mater 117 (2009) 1-9.

[4] M. Manzano, M. Colilla, M. Vallet-Regi, Exp. Opin. Drug Deliv 6 (2009) 1383-1400.

[5] E.V. Parfenyuk, B. Menaa, in: R. Morris (Ed.), Sol-Gel Process: Uniformity, Polymers 
and Applications, Nova Press Publishers, New York, 2010.

[6] J.M. Rosenholm, M. Lindén, J. Controll. Release 128 (2008) 157-164.

[7] S. Huh, J.W. Wiench, B.G. Trewyn, S. Song, M. Pruski, V.S.-Y. Lin, Chem. Commun. (2003) 2364-2365.

[8] W. Xu, Q. Gao, Y. Xu, D. Wu, Y. Sun, W. Shen, F. Deng, J. Solid State Chem. 181 (2008) 2837-2844.

[9] G. Wang, A.N. Otuonye, E.A. Blair, K. Denton, Zh. Tao, T. Asefa, J. Solid State Chem. 182 (2009) 1649-1660.

[10] M. Popova, A. Szegedi, I. Kolev, J. Mihály, B. Tzankov, G. Momekov, N. Lambov,

K. Yoncheva, Int. J. Pharm. 436 (2012) 778-785.

[11] J.C. Doadrio, E. Sousa, I. Izquierdo-Barba, A.L. Doadrio, J. Perez-Pariente, M. Vallet-Regi, J. Mater. Chem. 16 (2006) 462-466.

[12] M. Manzano, V. Aina, C.O. Arean, F. Balas, V. Cauda, M. Colilla, M.R. Delgado, M. Vallet-Regi, Chem. Eng. J. 137 (2008) 30-37.

[13] P. Horcajada, A. Ramia, G. Ferey, M. Vallet-Regi, Solid State Sci. 8 (2006) 1243-1249.

[14] Y.-J. Yang, X. Tao, Q. Hou, Y. Ma, X.-L. Chen, J.-F. Chen, Acta Biomater. 6 (2010) 3092-3100.

[15] R. Liu, P.H. Liao, J.K. Liu, P.Y. Feng, Langmuir 27 (2011) 3095-3099.

[16] S. Bhattacharyya, H. Wang, P. Ducheyne, Acta Biomater. 8 (2012) 3429-3435.

[17] J. Zheng, X. Tian, Y. Sun, D. Lu, W. Yang, Int. J. Pharm. 450 (2013) 296-303.

[18] J.M. Rosenholm, A. Meinander, E. Peuhu, R. Niemi, J.E. Eriksson, C. Sahlgren, M. Linden, ACS Nano 3 (2009) 197-206.

[19] H. Tang, J. Guo, Y. Sun, B. Chang, Q. Ren, W. Yang, Int. J. Pharm. 421 (2011) 388396.

[20] M. Ekkapongpisit, A. Giovia, C. Follo, G. Caputo, C. Isidoro, Int. J. Nanomed. 7 (2012) 4147-4158.

[21] L. Yuan, Q. Tang, D. Yang, J. Zhang, F. Zhang, J. Hu, J. Phys. Chem. 115 (2011) 9926-9932.

[22] J. Cosnes, C. Gower-Rousseau, P. Sekslk, A. Cortot, Gastroenterology 140 (2011) $1785-1794$.

[23] K. Dilger, M. Schwab, M.F. Fromm, Bowel Dis 10 (2004) 578-583.

[24] U. Klotz, M. Schwab, Adv. Drug Deliv. Rev 57 (2005) 267-279.

[25] B. Moulari, D. Pertuit, Y. Pellequer, A. Lamprecht, Biomaterials 29 (2008) 4554-4560.

[26] S. Huh, J.W. Wiench, J.-Ch Yoo, M. Pruski, V.S.-Y. Lin, Chem. Mater. 15 (2003) 4247-4256.

[27] G. Socrates, Infrared and Raman Characteristic Group Frequencies: Tables and Charts, 3rd ed., John Wiley and Sons, Ltd, Chichester, 2001.

[28] C. Song, W. Shi, H. Jiangt, J. Tu, D. Ge, J. Membr. Sci. 372 (2011) 340-345.

[29] J.D. Linehan, G. Kolios, V. Valatas, D.A. Robertson, J. Westwick, Free Radic Biol. Med. 39 (2005) 1560-1569.

[30] I. Chantret, A. Barbat, E. Dussaulx, M.G. Brattain, A. Zweibaum, Cancer Res. 48 
(1988) 1936-1942.

[31] H. Ohtsu, K. Yamauchi, O. Yoshie, Y. Tanno, H. Saito, N. Hayashi, T. Takishima, Cell Struct. Funct 18 (1993) 125-133.

[32] C.K. Wong, C.Y. Ho, C.W. Lam, J.P. Zhang, N.M. Hjelm, Immunol. Lett. 68 (1999) 317-323.

[33] Y. Cho, R. Shi, R. Borgens, A. Ivanisevic, Nanomedicine 3 (2008) 507-519. 